\title{
The relationship between rumination, depression and self-stigma in hazardous drinkers: an exploratory study
}

\section{Bojana Vilus}

Western Sydney University

Tania Perich ( $\nabla$ t.perich@westernsydney.edu.au )

Western Sydney University

\section{Short Report}

Keywords: self-stigma, alcohol use disorder, rumination, depression

Posted Date: August 26th, 2021

DOI: https://doi.org/10.21203/rs.3.rs-840687/v1

License: (c) (i) This work is licensed under a Creative Commons Attribution 4.0 International License.

Read Full License 


\section{Abstract}

Self-stigma may have an important impact on people living with alcohol use disorders, however, few studies have explored the relationship between rumination and depression on self-stigma for people with hazardous drinking. This study aimed to explore the relationship between rumination, self-stigma and depressive symptomatology for those with hazardous drinking levels and the relationship between these and alcohol use severity. Participants were recruited online between February and May of 2019 through paid advertising on a page created on Facebook by the researcher. Two hundred and one participants completed the online survey questionnaires, with 114 (56.7\%) meeting the Alcohol Use Disorders Identification Test (AUDIT) criteria with a score of 8 or above indicating hazardous drinking. Multiple regression analysis found that self-stigma was significant predictor of alcohol severity. Depression was not found to be a significant predictor of self-stigma; however, rumination was a significant predictor of self-stigma, explaining $36.7 \%$ of the variance. Although rumination was not a significant predictor of alcohol use severity in this study, it appeared to play an important part in the self-stigma for people engaged in hazardous drinking. More research is needed to determine the mediating factors in this relationship and the impact of these for people with hazardous drinking levels over time.

\section{Introduction}

Alcohol use disorder (AUD) is one of the most stigmatised mental disorders [1], with alcohol dependent individuals being held accountable for their disorder and viewed as not having a mental disorder at all [1]. By internalising negative public beliefs, individuals impacted by self-stigma may start to experience low self-esteem which leads to low self-efficacy and low confidence [2], with higher levels of self-stigma being found to be associated with depression, anxiety and lower quality of life in those with substance use disorders [3].

Previous studies have suggested that mood disorders may directly or indirectly influence alcohol use and vice versa, for instance, excessive alcohol consumption may contribute to a diagnosis of depression whilst people experiencing depression may also engage in excessive alcohol use. For example, one study found that their participants who had a substance use disorder (SUD) were up to $40 \%$ more likely to be diagnosed with depression, while the participants who had been diagnosed with depression were $3.5 \%$ likely to be diagnosed with SUD [4].

A common feature of mood disorders is rumination, where this cognitive style can increase vulnerability to depressive symptoms such as low mood, feelings of sadness and diminished energy [5]. However, there is evidence that rumination may be a transdiagnostic feature, underlying a range of internalising disorders, such as eating disorders like bulimia nervosa, as well as an array of physical illnesses such as chronic pain [6]. Rumination can directly or indirectly cause the cognitive experiences of cravings as the individual uses alcohol to cease the rumination process [7], with the likelihood of a relapse increasing due to alcohol dependent individuals' engagement in rumination [8]. Studies have shown that rumination can also increase the risk of alcohol use and dependency, independently of depression [9]. 
Self-stigma and rumination may be associated with increased depression and alcohol use independently, however currently there is a lack of research exploring the relationship between rumination and selfstigma among hazardous drinkers. This study aimed to explore the relationship between rumination, selfstigma, and depressive symptomatology among hazardous drinkers. It is hypothesised that higher levels of self-stigma will be associated with greater rumination, greater alcohol severity, as well as higher depression scores. It is further hypothesised that rumination, and depression, will significantly predict self-stigma scores, with depression being the greatest predictor. It is also expected that self-stigma will be predicted by higher scores on rumination and depression scales. Additionally, it is expected that greater alcohol severity will be predicted by higher rumination scores.

\section{Method}

\section{Participants}

Participant recruitment occurred online through paid advertising on a page created on Facebook by the researcher. To qualify for inclusion in this study, participants confirmed they were age 18 years or over and could understand English and meeting the Alcohol Use Disorders Identification Test (AUDIT) [10] criteria with a score of 8 or above indicating hazardous drinking. One hundred and fourteen participants' data were analysed with 35 males (30.7\%), 78 females (68.9\%), and one other gender identity $(0.9 \%)$. Participants ages ranged between 18 and 70 years $(M=33.5, S D=12.35)$. Table 1 displays demographic and clinical characteristics of the sample. 
Table 1

Demographic and clinical characteristics of sample

\begin{tabular}{|c|c|c|c|}
\hline & & $n$ & $\%$ \\
\hline \multirow[t]{3}{*}{ Sex } & Male & 35 & 17.4 \\
\hline & Female & 78 & 38.8 \\
\hline & Other & 1 & 0.5 \\
\hline \multirow[t]{3}{*}{ Marital status } & Married/Defacto & 28 & 13.9 \\
\hline & Never Married & 60 & 29.9 \\
\hline & Separated/Divorced/Widowed & 26 & 12.9 \\
\hline \multirow[t]{8}{*}{ Country of birth } & Australia/New Zealand & 27 & 13.4 \\
\hline & Europe & 1 & 0.5 \\
\hline & UK & 6 & 3 \\
\hline & Ireland & 24 & 11.9 \\
\hline & South Africa & 43 & 21.4 \\
\hline & Canada & 9 & 4.5 \\
\hline & Iran & 1 & 0.5 \\
\hline & USA & 3 & 1.5 \\
\hline \multirow[t]{3}{*}{ Occupational status } & Full-time & 52 & 46 \\
\hline & Part-time/Casual/Volunteer & 30 & 26.5 \\
\hline & Unemployed & 31 & 27.4 \\
\hline \multirow[t]{3}{*}{ Education } & Secondary/Completed Secondary & 45 & 40.7 \\
\hline & Bachelor or higher & 38 & 33.6 \\
\hline & Vocational qualification & 29 & 25.7 \\
\hline \multirow[t]{3}{*}{ Religious affiliation } & Religious & 51 & 25.4 \\
\hline & Non-religious & 59 & 29.4 \\
\hline & Unknown & 4 & 2 \\
\hline \multirow[t]{4}{*}{ Psychiatric diagnoses } & No diagnosis & 56 & 49.1 \\
\hline & Depression & 21 & 18.4 \\
\hline & Comorbid disorders & 31 & 27.2 \\
\hline & Other & 6 & 5.3 \\
\hline
\end{tabular}




\begin{tabular}{|llll|}
\hline & & $n$ & $\%$ \\
\hline Physical diagnoses & Yes & 41 & 20.4 \\
\hline Prior hospitalisation for psychiatric diagnoses & Yes & 21 & 10.5 \\
\hline Prescribed medication & Yes & 47 & 23.4 \\
\hline Non-prescribed drug use & None & 31 & 27.2 \\
& Marijuana & 45 & 39.5 \\
& Other & 38 & 33.3 \\
\hline Current alcohol treatment & Some treatment & 5 & 2.5 \\
\hline Prior alcohol treatment & Some treatment & 21 & 10.4 \\
\hline
\end{tabular}

\section{Measures}

Demographic questions included age, gender, relationship status, education, occupational status, religious affiliation, psychiatric diagnosis, alcohol treatment history, medical history, and current medication use.

\section{The Alcohol Use Disorders Identification Test - Self-report Version (AUDIT)}

The Alcohol Use Disorders Identification Test (AUDIT) is a screening scale that measures hazardous drinking levels with scores ranging from 0 to 4 per question and total scores ranging from 0 to 40 [10].The internal consistency for the AUDIT has been reported as high with a Cronbach's alpha $a=0.93$ ( $N$ $=1888)[10]$.

\section{Depression Anxiety and Stress Scale (DASS 21)}

The DASS 21 is a self-report measure with three subscales which measure depression, anxiety and stress [11]. The internal consistency for the DASS 21 has been reported as excellent with high temporal stability. Cronbach's alpha for the Depression subscale has been reported as $a=0.91$, [11]. The DASS-Depression subscale was used in this study.

\section{Ruminative Responses Scale (RRS)}

The Ruminative Responses Scale (RRS) is a 22-item scale was used to assess rumination [12]. The internal consistency for the RRS was reported as high with Cronbach's alpha for the scale $a=0.90(N=$ 1328) [12].

\section{Internalised Stigma of Mental IIIness Scale (ISMI)}


Internalised Stigma of Mental IIIness Scale (ISMI) is a 29-item scale that measures self-stigma for individuals with a mental illness. The internal consistency for ISMI was high with Cronbach's alpha for Alienation subscale $a=0.84$, for Stereotype Endorsement subscale $a=0.75$, for Discrimination Experience subscale $a=0.82$, for Social Withdrawal subscale $a=0.85$, and for Stigma Resistance subscale $a=0.53$ $(N=758)[13]$.

\section{Substance Use Stigma Mechanism Scale (SU-SMS) [14]}

The Substance Use Stigma Mechanism Scale (SU-SMS) is an 18-item scale which measures internalisedstigma, enacted and anticipated stigma among a substance using population. The internal consistency of the SU-SMS scale has been reported as significant with Cronbach's alpha $a=0.90$ across all the subscales $(N=175)$ [14]. The internalised-stigma subscale was utilised in this study.

\section{Procedure}

Participants accessed the survey via a link that was advertised on Facebook and the researcher's social media pages. Participants viewed a Participants Information Statement, which included an explanation of the purpose of this study, requirements of participants, researcher's contact details, and information about confidentiality. Those who selected "I consent to participate in this study" were taken to the survey, which was estimated to take 20 minutes to complete.

Contact details for crisis and mental health support services were provided in the Participant Information Statement and again at the conclusion of the survey. The study was approved b y Western Sydney University Human Research Ethics Committee (HREC Approval Number: H13034).

\section{Data Analyses}

Based on the statistical assumptions for the detection of a medium effect size utilising G-power analysis ( $f 2=0.05$ ) based upon a 0.90 power level at a significance level of 0.05 , a minimum target sample of 173 participants were required for mediation analysis. A frequency analysis revealed that 114 participants met the AUDIT criteria of scoring 8 and above. A one-way between-groups analysis of variance (ANOVA) was conducted to explore the impact of demographic characteristics on scores on depression (DASS 21 depression subscale), internalised stigma (SU-SMS - internalised stigma subscale), self-stigma (ISMI) and rumination (RRS).

\section{Results}

An ANOVA was conducted on demographics variables of age, gender, and education status $(N=114)$, and the DASS-Depression subscale $(M=10.59, S D=5.56)$, self-stigma (ISMI) $(M=2.07, S D=.49)$, internalised stigma (SU-SMS) $(M=14.60, S D=7.27)$ and rumination $(M=54.72, S D=15.47)$. No significant differences were found. 
A Pearson product-moment correlation coefficient analysis was conducted to assess the relationship between AUDIT total score, internalised stigma (SU-SMS), self-stigma (ISMI), rumination (RRS), and depression (DASS 21). A medium positive correlation was found between AUDIT total score and internalised stigma subscale (SU-SMS) $r=0.45, \mathrm{n}=114, p<.001$ with higher scores on the internalised stigma subscale being associated with higher scores on the AUDIT scale. A further strong positive correlation was found between rumination and depression $r=0.49, \mathrm{n}=114, p<.001$ with higher scores on the rumination scale being associated with higher scores on the depression subscale.

A multiple regression analysis was conducted to assess the ability of self-stigma as measured by (ISMI and SU-SMS-Internalised Stigma subscale), depression (DASS 21), and rumination (RRS) to predict the levels of total AUDIT scores. A significant regression equation was found $F(4,106)=8.38, p<.000, \mathrm{R}^{2}$ .240 , with SU-SMS Internalised Stigma subscale significantly predicting $24 \%$ of the variance of the AUDIT score. Depression, ISMI and Rumination did not significantly explain the variance of the AUDIT scores and were excluded from the analysis.

A further multiple regression analysis was conducted to assess the ability of rumination as measured by (RRS) to significantly predict self-stigma scores as measured by (ISMI) total score. A significant regression equation was found $F(1,110)=63.79, p<.000, \mathrm{R}^{2} .367$, with $36.7 \%$ of the variance being explained by this measure.

\section{Discussion}

This is one of the first studies to explore the relationship between rumination and self-stigma among a hazardous drinking population. Consistent with previous research [1] and with the hypothesis of this study, self-stigma and internalised stigma were found to be significant predictors of alcohol use severity. Like previous research [1], this study may support the idea that self-stigma is associated with greater hazardous drinking.

This study further hypothesised that high scores on the self-stigma scales would be associated with high scores on the rumination scale and that high scores on the self-stigma scales would be associated with greater depression scores. Here rumination scores were found to be significantly correlated with depression scores, as has been found in previous research [15], and self-stigma was found to be associated with greater depression scores. Additionally, rumination was found to be a significant predictor of self-stigma in the hazardous drinking sample, which is a novel finding of this study. This may indicate areas that could potentially be addressed in psychological interventions targeting transdiagnostic features in substance use.

Limitations of this study include the use of self-report data, whilst it is also possible that some may have under reported their diagnoses or alcohol usage due to the stigma surrounding mental illness. The participants were asked to answer questions regarding any experiences of internalised stigma, as measured by ISMI however, it is possible that the participants did not deem alcohol dependency as a mental disorder, therefore they did not report internalised stigma for their alcohol use habits specifically. 
Rumination was found to be important in understanding self-stigma for those engaged in hazardous drinking behaviours. Self-stigma significantly predicted alcohol use severity, and in addition, those who scored higher on the rumination scale also scored higher on the self-stigma scales. Depression was not found to be a significant predictor, suggesting that rumination is a unique contributor to the experiences of self-stigma for this group. More research is needed to the impact of these on drinking behaviours over time and whether these features should be addressed in transdiagnostic psychological interventions addressing hazardous drinking.

\section{Declarations}

\section{Data Availability Statement}

The data that support the findings of this study are available from the corresponding author upon request.

\section{References}

1. Schomerus G, Lucht M, Holzinger A, Matschinger H, Carta MG, Angermeyer MC. The Stigma of Alcohol Dependence Compared with Other Mental Disorders: A Review of Population Studies. Alcohol and Alcoholism. 2010;46(2):105-12. https://doi.org/10.1093/alcalc/agq089

2. Larson JE, Corrigan PW. Psychotherapy for self-stigma among rural clients. Journal of Clinical Psychology. 2010;66(5):524-36. https://doi.org/10.1002/jclp.20679

3. Brown SA, Kramer K, Lewno B, Dumas L, Sacchetti G, Powell E. Correlates of Self-Stigma among Individuals with Substance Use Problems. International Journal of Mental Health and Addiction. 2015;13(6):687-98. https://doi.org/10.1007/s11469-015-9559-9

4. Grant BF, Goldstein RB, Saha TD, Chou SP, Jung J, Zhang H, et al. Epidemiology of DSM-5 Alcohol Use Disorder: Results From the National Epidemiologic Survey on Alcohol and Related Conditions III. JAMA Psychiatry. 2015;72(8):757-66. https://doi.org/10.1001/jamapsychiatry.2015.0584

5. Nolen-Hoeksema S, Morrow J. Effects of rumination and distraction on naturally occurring depressed mood. Cognition and Emotion. 1993;7(6):561-70. https://doi.org/10.1080/02699939308409206

6. Sansone RA, Sansone LA. Rumination: relationships with physical health. Innovations in clinical neuroscience. 2012;9(2):29. https://doi.org/10.1111/j.1360-0443.1993.tb02093.x

7. Spada MM, Caselli G, Wells A. A Triphasic Metacognitive Formulation of Problem Drinking. Clinical Psychology \& Psychotherapy. 2013;20(6):494-500. https://doi.org/10.1002/cpp.1791

8. Caselli G, Gemelli A, Querci S, Lugli AM, Canfora F, Annovi C, et al. The effect of rumination on craving across the continuum of drinking behaviour. Addictive Behaviors. 2013;38(12):2879-83. https://doi.org/10.1080/16066350802100822

9. Caselli G, Bortolai C, Leoni M, Rovetto F, Spada MM. Rumination in problem drinkers. Addiction Research \& Theory. 2008;16(6):564-71. 
10. Saunders JB, Aasland OG, Babor TF, De La Fuente JR, Grant M. Development of the Alcohol Use Disorders Identification Test (AUDIT): WHO Collaborative Project on Early Detection of Persons with Harmful Alcohol Consumption-II. Addiction. 1993;88(6):791-804. https://doi.org/10.1111/j.13600443.1993.tb02093.x

11. Lovibond PF, Lovibond SH. The structure of negative emotional states: Comparison of the Depression Anxiety Stress Scales (DASS) with the Beck Depression and Anxiety Inventories. Behaviour Research and Therapy. 1995;33(3):335-43. https://doi.org/10.1016/00057967(94)00075-U

12. Treynor W, Gonzalez R, Nolen-Hoeksema S. Rumination Reconsidered: A Psychometric Analysis. Cognitive Therapy and Research. 2003;27(3):247-59. https://doi.org/10.1023/a:1023910315561

13. Hammer JH, Toland MD. Internal structure and reliability of the Internalized Stigma of Mental Illness Scale (ISMI-29) and Brief Versions (ISMI-10, ISMI-9) among Americans with depression. Stigma and Health. 2017;2(3):159-74. https://doi.org/10.1037/sah0000049

14. Smith LR, Earnshaw VA, Copenhaver MM, Cunningham CO. Substance use stigma: Reliability and validity of a theory-based scale for substance-using populations. Drug and Alcohol Dependence. 2016;162:34-43. https://doi.org/10.1016/j.drugalcdep.2016.02.019

15. Nolen-Hoeksema S, Stice E, Wade E, Bohon C. Reciprocal relations between rumination and bulimic, substance abuse, and depressive symptoms in female adolescents. Journal of Abnormal Psychology. 2007;116(1):198-207. https://doi.org/10.1037/0021-843X.116.1.198 